\title{
CHANGING TRENDS IN PATTERN OF PRESENTATION WITH DIFFERENT TYPES OF PYREXIA AND APPROACH TO BE MADE
}

\author{
PROF. KHAN ABUL KALAM AZAD \\ Editor, Journal of Dhaka Medical College
}

J Dhaka Med Coll. 2011; 20(1) : 1-3.

With increasing number of patients with pyrexia, doctors are often faced with the diagnostic dilemma of a febrile patient who has different types of manifestations. Fever is an important and common presentation of tropical diseases and may sometimes be the only presentation of a serious illness. Diagnosis is often difficult because of the many diagnostic possibilities, symptoms and signs which may often be non-specific and because of doctors' unfamiliarity with many tropical diseases. Fever is a manifestation of an illness but not an illness itself. By far, the most common cause of fever is infection. However, other causes of fever should always be kept in mind, such as malignancy (especially lymphoma and leukaemia), connective tissue diseases, drugs, pulmonary embolism and other less common causes.

Fever patterns are rarely diagnostic but can be helpful in certain infections. As for example, Dengue virus infection typically presents with two febrile periods separated by an afebrile interval of one to three days (saddle back fever). Fevers that occur at regular intervals of 42-78 hours are virtually pathognomic of P.vivax, P.ovale, P.malariae infections. Although untreated typhoid fever classically presents with a continued fever associated with relative bradycardia, almost any fever pattern can develop. Nowadays the pattern of fever is changing. The common associated presentations are also changing which include myalgia, headache, backache, pain in other site of the body, vomiting, cough, diarrhoea, runny nose, arthralgia, burning sensation of micturition, different types of rashes. Many infectious diseases have common and overlapping clinical features. Due to emergence of many new infectious diseases e.g.viral haemorrhagic fever, yellow fever Chikungunya, Nipah encephalitis pattern of fever is changing. Besides the same infectious agent can also change presentation of clinical features after a time interval, the most common example is Dengue hemorrhagic fever. Now dengue is commonly associated with liver and renal function impairment and associated respiratory tract infection which was very uncommon in previous years.

Continued fevers are those that are constant with only slight remissions and can be seen in such diseases as lobar pneumonia and rickettsial disease. An intermittent fever is one with wide fluctuations, usually normal in the morning and peaking in the afternoon or evening. This fever pattern can be seen in many viral diseases as well as endocarditis or localized pyogenic infections such as an abscess. A diurnal pattern also known as typhus inversus, is the reverse of normal circadian pattern in which the highest temperature is in the morning. It can be found in milliary TB, hepatic abscesses and endocarditis. Saddleback or biphasic fevers are those that are constant for several days, spontaneously reduce for 1 to 2 days, and then increase again. Saddleback fevers can be seen in such infections as dengue, yellow fever and influenza. A Pel-Ebstein fever is one that occurs daily for a period of about one week, resolves for about an equal time, and then recurs. Pel-Ebstein fevers are often seen in lymphomas. The Jarisch-Herxheimer reaction 
is a fever (and sometimes more systemic symptoms such as hypotension) that occurs several hours after treatment with antibiotics. It is a classic reaction for syphilis, but can also be seen in leptospirosis, relapsing fever and brucellosis.

A history of specific exposure should be sought from the patients. Eliciting unique exposure may help to point to a specific diagnosis. For example, a patient with a history of tick bite presenting with fever, rash, regional lymphadenopathy, and a painless skin eschar suggests tick typhus due to rickettsia. Traditional cultural restraints on sexual behavior may not be present during travel, and therefore a history of sexual exposure is important when evaluating the febrile traveler. A history of unprotected sexual intercourse, for example, may point to the diagnosis of an acute retroviral syndrome with HIV, which often manifests as fever and mononucleosis like illness one to six weeks after exposure. As many tropical diseases have overlapping and non-specific signs and symptoms, a unique exposure is often the only clue to correct diagnosis.

A thorough physical exam is very important in the diagnosis of febrile illnesses and should be repeated during the course of illness to evaluate for changes. While the exam is often directed by symptoms, special attention should be paid to the skin, lymph nodes, eyes, nail beds, cardiovascular system, chest, abdomen, joints and neurological system. A genital or pelvic exam and rectal exam should be considered as a part of complete evaluation.

Symptoms and physical findings should guide the laboratory evaluation. Often, no lab studies are required to make the diagnosis of a simple viral illness. When the diagnosis is not clear-

- A complete blood count should almost always be ordered, with special attention paid to the differential. The presence of increased neutrophils and band forms (as well as toxic granulations and Dohle bodies) may indicate a bacterial infection, while neutropenia might be seen in viral infections or infiltrative process of the bone marrow such as malignancy, TB, or histoplasmosis. Lymphocytosis may be seen in viral infections, typhoid fever, brucellosis, or TB, and atypical lymphocytes are frequently seen in infections with Epstein-Barr virus, cytomegalovirus or HIV. Monocytosis may be seen with typhoid fever, TB, or lymphoma. Eosinophilia can be seen with certain parasitic infestations, a hypersensitivity drug reaction, or lymphoma.

- Urine analysis with examination of the urinary sediment is indicated when symptoms suggest involvement of the genitourinary system or when another exclusive diagnosis is not really apparent.

- Electrolytes, glucose, BUN, and creatinine should be checked as a general measure of the patient's condition. Liver function tests are also generally indicated if other signs and symptoms do not specifically point away from hepatic involvement.

- Smears and cultures of specimens from the throat, urethra, anus, cervix, or vagina should be obtained when symptoms or signs indicate involvement of those systems. Cultures of blood and urine are indicated whenever the illness is thought to be more than a simple viral infection, especially if the diagnosis is unclear and empiric antibiotics are being considered.

- Cerebrospinal fluid should be examined and cultured if there is an associated severe headache, meningismus, a change in mental status. If signs of increased intracranial pressure, such as focal neurological deficit or papilloedema, are present, a CT of the head should be performed to rule out a mass lesion before performing a lumber puncture.

- Any abnormal fluid collection (pleural, peritoneal or joint) found in the presence of fever should be examined and cultured.

- if a fever is severe or prolonged, it is appropriate to order an erythrocyte sedimentation rate $(\mathrm{ESR})$ or $\mathrm{C}$ - reactive 
protein (CRP) as a baseline. These tests are too non-specific to use for diagnostic purposes, but can be used to follow the patient's progress.

- A chest X-ray should be considered standard in any patient with an unclear diagnosis or if signs and symptoms point to the chest. $\mathrm{X}$-rays of other areas, such as the abdomen or an extremity, may be indicated by the patient's symptoms.

- A CT scan abdomen, CT chest or MRI brain may also be considered according to site of involvement and suspicious lesion.

A stepwise and rational approach to febrile patients will allow for correct diagnosis and optimal management in most patients. When evaluating fever in a tropical country, the doctor must first determine if the patient has a tropical disease or any other illness. If a tropical infection is suspected doctors should consider which illnesses are potentially fatal, which are treatable and which infections are potentially transmissible and may therefore pose a public health risk. Such an approach needs a clear understanding of tropical illnesses and their presentation and epidemiological background. A working differential diagnosis can be formulated from a complete history, physical examination, knowledge of the most common diseases affecting travelers and of the incubation periods of these diseases, and appropriate laboratory investigations.

\section{References:}

1 Fortnightly Review: Evaluating fever in travellers returning from tropical countries Atul Humar, fellow in infectious diseases, Jay Keystone (jay.keystone@utoronto.ca), professor of medicine Author Affiliations a Tropical Disease Unit, Division of Infectious Diseases, Department of Medicine, Toronto Hospital, University of Toronto, ENG212, 200 Elizabeth Street, Toronto, Ontario M5G 2C4

2. Operational Medicine 2001 Health Care in Military Settings, The Brookside Associates Medical Education Division.

3. Fever: Basic Mechanisms and Management, Second Edition. Edited by Philip A Mackowiak. Lippincott-Raven Publishers, Philadelphia.

4. Gelfand JA and Dinarello CA. Fever and Hyperthermia. Harrison's Principles of Internal Medicine, 14th edition. Edited by Anthony Fauci, et al. McGraw-Hill Companies, Inc. USA, 1998: 84-89. 5.Mackowiak PA, et al. Concepts of fever: recent advances and lingering dogma. Clinical Infectious Diseases 1997; 25:119-138.

6. A Comprehensive Evidence-Based Approach to Fever of Unknown Origin Ophyr Mourad, MD, FRCPC; Valerie Palda, MD, MSc; Allan S. Detsky, $\mathrm{MD}, \mathrm{PhD}$

7. Etiology and Outcome of Fever After a Stay in the Tropics Emmanuel Bottieau, MD; Jan Clerinx, MD; Ward Schrooten, MD, PhD; Erwin Van den Enden, MD; Raymond Wouters, MD; Marjan Van Esbroeck, MD; Tony Vervoort, MD; Hendrik Demey, MD; Robert Colebunders, MD, PhD; Alfons Van Gompel, MD; Jef Van den Ende, MD, PhD

8. Changing patterns of communicable disease in England and Wales Part ]-Newly recognised diseases N S GALBRAITH, PAT FORBES, R T MAYON-WHITE 